\title{
ESTUDIO DE LAS CAMPAÑAS DE VACUNACION ANTIGRIPAL 2005 Y 2006 A TRABAJADORES SANITARIOS DEL AREA 2 DE MADRID
}

\author{
B. PÉREZ BERMÚDEZ*, M. GARCÍA CEBRIÁN*, Mª J. MARTÍNEZ DE ARAMAYONA*, \\ A. B. NIETO SÁNCHEZ**, C. RAMOS ARGÜELLO**, A. ARANDA VIZCAÍNO**. \\ (*) Medico/a de Trabajo \\ (**) Enfermero/a del Trabajo. \\ Servicio de Prevención de Riesgos Laborales del área 2. Madrid. Hospital de la Princesa.
}

\section{RESUMEN}

La vacunación antigripal es la medida más eficaz para prevenir la gripe. Dentro de los colectivos laborales en los que se recomienda su inoculación, se encuentran los trabajadores sanitarios.

Objetivos:, Estudiar si existen diferencias significativas entre las Campañas del 2005-06 y 2006-07 dirigidas a los trabajadores del área sanitaria 2 de la Comunidad de Madrid.

Metodología: Estudio descriptivo de ambas campañas en el área sanitaria 2 de la Comunidad de Madrid. Se realiza un análisis univariante y se construye un modelo de Regresión Logística cuya variable dependiente son las dos campañas. Como variables independientes se incluyeron: edad, categoría, servicio, centro de trabajo, vacunación de la gripe en el año anterior, efectos secundarios en año el anterior, enfermedad crónica, medicación, fumador y procesos alérgicos.

Resultado:. En la Campaña 2005 se vacunaron más en servicios centrales, hostelería y administración. Se declararon más efectos secundarios, se vacunaron más sanitarios y más trabajadores declararon tomar algún tipo de medicación. Sin embargo en la Campaña 2006 se vacunaron más en el H. U. Princesa, en la primera semana de campaña, en los servicios médicos y más trabajadores declararon tener algún tipo de enfermedad crónica.

Conclusiones: Las coberturas vacunales han sido iguales o inferiores a años anteriores en los distintos centros excepto en el Hospital U. de la Princesa donde fue mayor.

\section{PALABRAS CLAVES}

Vacuna de la gripe en trabajadores sanitarios, Comparación de Campañas de vacunación de la gripe.

\section{ABSTRACT}

The vaccination against the influenza is the most effective way of preventing this illness. Workers in the Sanitary sector are ones of the most recommended to be vaccinated against the flu.

Objectives: To study whether there are significant differences between the 2005-2006 and 2006-2007 Campaigns addressed to the workers in the Sanitary area $n^{\circ} 2$ in the Madrid Community.

Methodology: Descriptive study of both campaigns in the Sanitary area $\mathrm{n}^{\circ} 2$ in the Madrid Community. A uni-variant analysis is carried out to build a Logistic Regression model in which the two campaigns are the dependant variables. The following independent variables were also included: age, professional category, service, working center, previous year anti-flu vaccination, side effects in previous year, chronic diseases, medication taken, smoking habits and allergic processes.

Results: In the 2005 campaign, vaccination was more intense in central services, catering and administration. There were more side effects, more sanitary workers were vaccinated and more workers declared to have taken any sort of medication. However, in the 2006 campaign there were more vaccinations in the "H.U. la Princesa", within the first week of campaign, in the medical services, and more workers declared to suffer any type of chronic disease.

Conclusions: The coverage of the vaccination has been equal or lower than in previous years in all working centers, with the exception of the "H.U. la Princesa", where the coverage was higher.

\section{KEY WORDS}

Influenza vaccination in sanitary workers. Comparison between anti-flu vaccination campaigns. 


\section{INTRODUCCION}

Todos los años la Consejería de Sanidad de Madrid por medio de la Dirección General de Salud Pública distribuye a los distintos centros sanitarios las vacunas frente a la gripe necesarias para la vacunación de los trabajadores de dichos centros $(1,2)$.

Desde el año 2005, en el Hospital Universitario de la Princesa de Madrid, las campañas vacunales frente a la gripe se planifican y se llevan a cabo íntegramente desde la Unidad de Vigilancia de la Salud del Servicio de Prevención de Riesgos Laborales del Área 2 y van dirigidas a todos los trabajadores de dicha área sanitaria.

La gripe es una infección viral causada por el Influenzavirus, generalmente leve y autolimitada, que cursa en forma de epidemias anuales. Supone un importante problema de salud pública tanto por la mortalidad como por los altos costes socioeconómicos secundarios.

La vacunación antigripal es la medida básica y más eficaz para prevenir la gripe y reducir el impacto de la epidemia (evidencia Ia). Se recomienda en mayores de 60 años, personas con enfermedades crónicas, como por ejemplo hipertensión arterial, diabetes mellitus, neuropatías, cardiopatías, enfermedades que causan inmunodepresión y aquellas que están en tratamientos con fármacos inmunosupresores, también a trabajadores de centros sanitarios y a otros colectivos como por ejemplo policías, bomberos, etc. $(3,4,5)$

\section{MATERIAL Y METODOS}

Estudio Descriptivo de las Campañas vacunales 2005-06 y 2006-07.

La vacuna inoculada en el año 2005 estaba constituida por las cepas: A/New Caledonia/20/99 (H1N1), A/California/7/2004(H3N2), B/Shanghai/361/2002 y en el año 2006: A/New Caledonia/20/99 (H1N1), de la cepa similar a A/Wisconsin/67/2005 (H3N2) y de la B/Malaysia/2506/2004. (6)

Las campañas de vacunación no se efectuaron en el mismo periodo de tiempo, en el año 2005 se realizó en los meses de octubre y noviembre, mientras que en el año 2006, comenzó más tarde, última semana de octubre, noviembre y diciembre.

Los trabajadores que decidían vacunarse, acudían a la unidad de vigilancia de la Salud. Sin embargo en el H. U. de la Princesa, en la campaña del 2006, las unidades de Salud Laboral fueron a los puestos de trabajo para facilitar su inoculación.

La técnica de vacunación era similar, intramuscular profunda preferiblemente en el deltoides.

En el acto de vacunación, se ofrecía al trabajador un breve cuestionario a cumplimentar voluntariamente, recogiendo las siguientes variables: edad, categoría, servicio, centro de trabajo, vacunación de la gripe en año anterior, efectos secundarios en año anterior, enfermedad crónica, medicación, fumador y procesos alérgicos.

Para la comparación de las variables categóricas, se utiliza el ji cuadrado y para las variables continuas, la t-student.

Como análisis multivariante, se construye un modelo de regresión logística no condicional, para detectar variables confusoras. La variable dependiente son las dos campañas, 2005 y 2006. Como medida de asociación se utiliza la odds ratio (O.R.) y se calculan sus Intervalos de Confianza (IC.) al $95 \%$.

\section{RESULTADOS}

En la campaña 2005 se inocularon 1722 dosis, de las cuales 81 eran a familiares de los trabajadores y en el 2006, 1957 dosis, siendo 197 inoculadas a familiares .

Como puede observarse en la tabla I, la cobertura vacunal en cada uno de los centros de trabajo ha sido similar o ha disminuido respecto a la campaña del 2005, excepto en el H. U. de la Princesa cuya cobertura ha sido cercana al $50 \%$.

En ambas campañas se observa una distribución desigual en la demanda de las vacunas por parte de los trabajadores, siendo en las primeras 4 semanas (grafico I) muy alta y disminuyendo en las siguientes.

La tabla II, recoge un resumen de las principales variables a estudio, comparando ambas campañas. Significación $\mathrm{p}<0,05$.

Las variables que han resultado significativas respecto a una u otra campaña, son: semanas de campaña, si son trabajadores sanitarios, servicios hospitalarios, si se han vacunado el año anterior, si padecían enfermedad crónica y si recibían medicación. 
Tabla I. Cobertura vacunal en los Centros sanitarios dependientes del Área 2.

\begin{tabular}{|c|c|c|}
\hline CENTROS & AÑO 2005 & AÑO 2006 \\
\hline H. U. DE LA PRINCESA & $35.1 \%$ (742 dosis) & $47.8 \%$ (1005 dosis ) \\
\hline H. SANTA CRISTINA & $17.5 \%$ (158 dosis) & $17.5 \%$ (158 dosis ) \\
\hline H. U. NIÑO JESUS & $30.6 \%$ (337 dosis) & $26.9 \%$ (296 dosis) \\
\hline C. E. HNOS. GCIA NOBLEJAS & $32.2 \%$ (38 dosis) & $23.7 \%$ (28 dosis) \\
\hline C. E. JAIME VERA & $50.0 \%$ (44 dosis) & $38.6 \%$ (34 dosis ) \\
\hline SUMMA & $10.1 \%$ (176 dosis) & $10.1 \%$ (176 dosis) \\
\hline LAVANDERIA & $21.1 \%$ (85 dosis ) & $15.5 \%$ (62 dosis) \\
\hline
\end{tabular}

Figura 1. Dosis Inoculadas durante las semana de Campaña vacunal

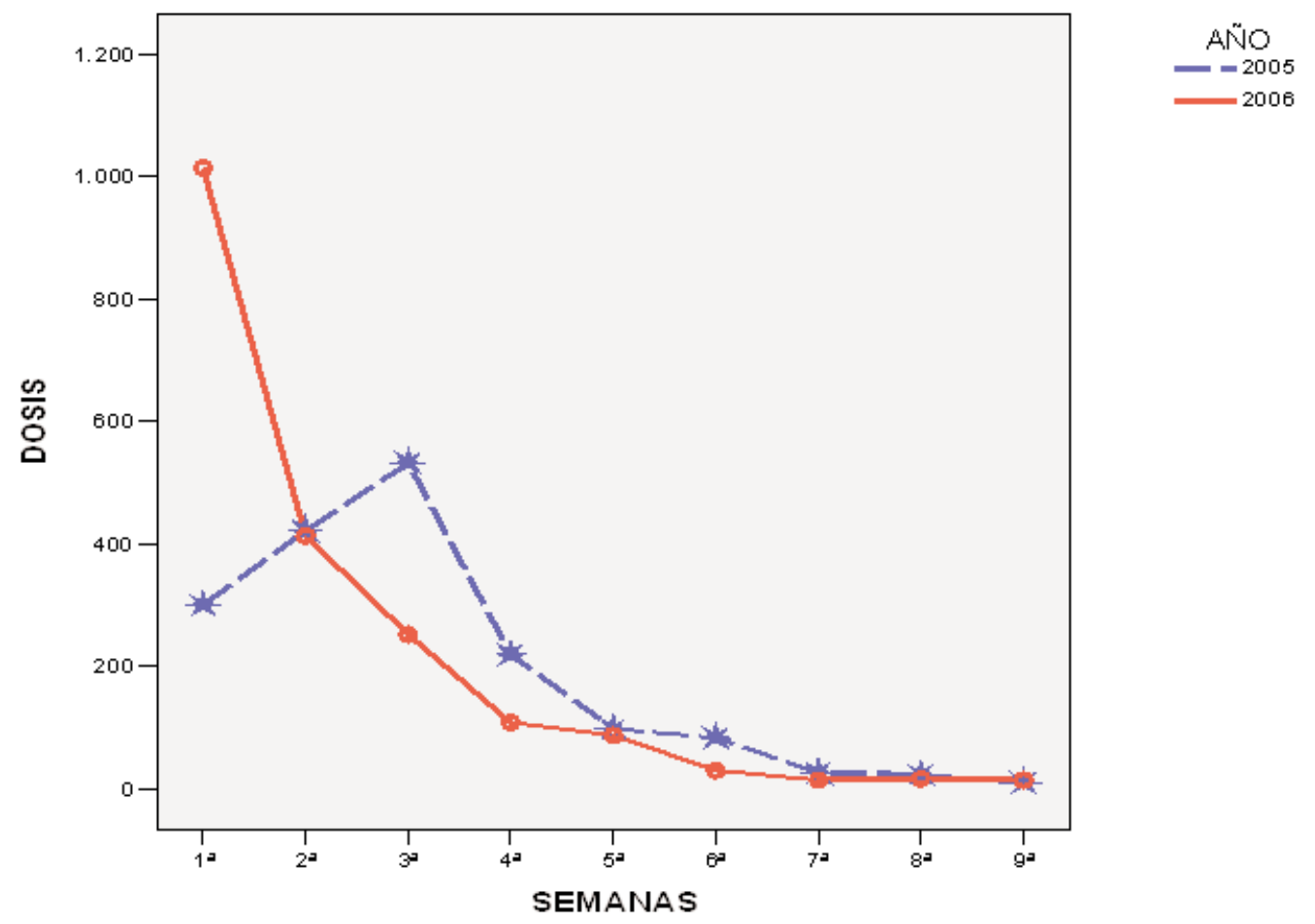

Respecto a los trabajadores, los sanitarios se vacunaron en una proporción mayor en el año 2005 que en el 2006. Los Servicios más vacunados en el año 2006 han sido las especialidades médicas y urgencias.
La proporción de vacunados el año anterior a la campaña ha sido mayor en el 2006. Por otra parte la prevalencia de trabajadores que declaran tomar medicación ha sido mayor en el 2005. Sin embargo en el 2006, son más los trabajadores que declaraban algún tipo de patología crónica. 
Tabla II. Descriptivo de las variables estudiadas.

\begin{tabular}{|c|c|c|c|}
\hline VARIABLE & AÑO 2005 & AÑO 2006 & $\mathbf{p}$ \\
\hline SEMANA DE CAMPAÑA & & & 0.000 \\
\hline Semana $1^{a}$ & $18.3 \%$ & $53.4 \%$ & \\
\hline Semana $2^{a}$ & $25.4 \%$ & $20.1 \%$ & \\
\hline Semana $3^{\text {a }}$ & $30.9 \%$ & $13.0 \%$ & \\
\hline Semana $4^{\text {a }}$ & $12.0 \%$ & $5.4 \%$ & \\
\hline Semana $5^{\text {a }}$ & $5.6 \%$ & $4.4 \%$ & \\
\hline Semana $6^{a}$ & $4.9 \%$ & $1.3 \%$ & \\
\hline Semana $7^{\mathrm{a}}$ & $1.4 \%$ & $0.7 \%$ & \\
\hline Semana $8^{a}$ & $0.9 \%$ & $1.0 \%$ & \\
\hline Semana 9 a & $0.5 \%$ & $0.7 \%$ & \\
\hline SEXO & & & 0.908 \\
\hline Mujeres & $71.4 \%$ & $71.6 \%$ & \\
\hline SANITARIOS & & & 0.011 \\
\hline Trabajadores Sanitarios & $61.6 \%$ & $57.2 \%$ & \\
\hline Trabajadores no sanitarios & $38.4 \%$ & $42.8 \%$ & \\
\hline SERVICIOS & & & 0.018 \\
\hline Medicas & $29.1 \%$ & $33.3 \%$ & \\
\hline Quirúrgicas & $13.1 \%$ & $11.5 \%$ & \\
\hline S. Centrales & $20.8 \%$ & $19.5 \%$ & \\
\hline Urgencias & $13.7 \%$ & $15.0 \%$ & \\
\hline Administración & $9.2 \%$ & $7.7 \%$ & \\
\hline Hostelería & $9.4 \%$ & $7.3 \%$ & \\
\hline Otros & $4.7 \%$ & $5.7 \%$ & \\
\hline VACUNACION EL AÑO ANTERIOR & & & 0.000 \\
\hline Si vacunado & $69.1 \%$ & $77.6 \%$ & \\
\hline No vacunado & $30.9 \%$ & $22.4 \%$ & \\
\hline EFECTOS SECUNDARIOS & & & 0.098 \\
\hline $\mathrm{Si}$ & $10.1 \%$ & $8.2 \%$ & \\
\hline No & $89.9 \%$ & $91.8 \%$ & \\
\hline ANTECEDENTES DE ENFERMEDAD & & & 0.002 \\
\hline $\mathrm{Si}$ & $19.0 \%$ & $23.5 \%$ & \\
\hline No & $81.0 \%$ & $76.5 \%$ & \\
\hline ALERGIA & & & 1.000 \\
\hline $\mathrm{Si} \quad$ & $20.1 \%$ & $20.1 \%$ & \\
\hline No & $79.9 \%$ & $79.9 \%$ & \\
\hline FUMAR & & & 0.247 \\
\hline $\mathrm{Si}$ & $27.6 \%$ & $25.7 \%$ & \\
\hline No & $72.4 \%$ & $74.3 \%$ & \\
\hline MEDICACION & & & 0.040 \\
\hline $\mathrm{Si}$ & $30.5 \%$ & $27.1 \%$ & \\
\hline No & $69.5 \%$ & $72.9 \%$ & \\
\hline CATEGORIAS & & & 0.250 \\
\hline MEDICO & $382(24.2 \%)$ & $456(25.9 \%)$ & \\
\hline ENFERMERO/A & $294(18.6 \%)$ & $297(16.9 \%)$ & \\
\hline AUX. ENFERMERIA & $224(14.2 \%)$ & $254(14.4 \%)$ & \\
\hline TECNICOS & $73(4.6 \%)$ & $116(6.6 \%)$ & \\
\hline CELADORES & $141(8.9 \%)$ & $139(7.9 \%)$ & \\
\hline ADMINISTRATIVO & $180(11.4 \%)$ & $173(9.8 \%)$ & \\
\hline CONDUCTORES & $24(1.5 \%)$ & $23(1.3 \%)$ & \\
\hline MANTENIMIENTO & $40(2.5 \%)$ & $34(1.9 \%)$ & \\
\hline LIMPIADORA & $34(2.2 \%)$ & $42(2.4 \%)$ & \\
\hline ESTUDIANTES & $60(3.8 \%)$ & $112(6.4 \%)$ & \\
\hline COCINAS & $32(2.0 \%)$ & $40(2.3 \%)$ & \\
\hline OTROS & $96(6.1 \%)$ & $73(4.2 \%)$ & \\
\hline$E D A D$ (años) & $43.1(12.4)$ * & $42.6(13.1)$ * & 0.250 \\
\hline
\end{tabular}

* = Media (Desviación típica) 
En el estudio multivariante (Tabla II), para la construcción del modelo de regresión logística no se ha introducido la variable categorías ya que suponía un problema de colinealidad con la variable sanitarios. El área de la curva R.O.C. es de 76.8 .
Las variables significativas coinciden con el análisis univariante, excepto que en el modelo construido, los trabajadores que referían efectos secundarios a la vacuna de la gripe el año anterior, son más prevalentes en el año 2005.

Tabla III. Regresión logística.

\begin{tabular}{|c|c|c|c|c|}
\hline & \multirow{2}{*}{$\begin{array}{c}\text { SIGNIF. } \\
\mathrm{p}\end{array}$} & \multirow[t]{2}{*}{ O. R } & \multicolumn{2}{|c|}{ IC AL 95,0\% } \\
\hline & & & INFERIOR & SUPERIOR \\
\hline $\begin{array}{l}\text { SEMANAS DE } \\
\text { VACUNACION }\end{array}$ & & & & \\
\hline SEMANA 1 & & 1 & & \\
\hline SEMANA 2 & ,000 & 286 & 228 & ,359 \\
\hline SEMANA 3 & ,000 & 147 & 114 & 189 \\
\hline SEMANA 4 &, 000 & ,172 & ,125 & ,237 \\
\hline SEMANA 5 & ,000 & ,316 & 209 & 479 \\
\hline SEMANA 6 &, 000 & ,073 &, 040 & ,134 \\
\hline SEMANA 7 &, 000 & ,144 &, 060 & ,343 \\
\hline SEMANA 8 & ,067 & ,427 & 172 & 1,062 \\
\hline SEMANA 9 & ,284 &, 555 & , 189 & 1,630 \\
\hline SEXO & ,765 & 970 & ,794 & 1,185 \\
\hline$E D A D$ &, 061 & ,993 & ,984 & 1,000 \\
\hline SERVICIOS & & & & \\
\hline MEDICAS & & 1 & & \\
\hline QUIRURGICAS & ,615 & ,926 & 686 & 1,250 \\
\hline CENTRALES & ,015 & ,730 &, 566 & ,940 \\
\hline URGENCIAS &, 552 & 915 & ,682 & 1,226 \\
\hline ADMINISTRA. &, 000 &, 507 & ,348 & ,739 \\
\hline HOSTELERIA &, 003 &, 540 & ,357 & ,816 \\
\hline OTROS & ,782 & ,937 &, 589 & 1,489 \\
\hline VACUNACION ANTERIOR &, 000 & 2,604 & 2,036 & 3,328 \\
\hline EFECTOS $2^{\circ}$ & ,042 &, 730 & ,538 & ,989 \\
\hline ENFERMEDAD CRONICA &, 000 & 1,817 & 1,408 & 2,344 \\
\hline$H^{a}$ DE ALERGIAS & ,165 & ,856 & ,687 & 1,066 \\
\hline FUMADOR & ,745 & ,967 & ,815 & 1,185 \\
\hline TOMA MEDICACION &, 000 & ,663 &, 527 & ,834 \\
\hline SANITARIOS & ,000 & 614 & ,480 & ,783 \\
\hline
\end{tabular}




\section{DISCUSIÓN}

Llama la atención, la masiva vacunación $(53.4 \%$ de las dosis ) durante la primera semana del año 2006, esto podría ser debido al retraso en la distribución de la vacuna por parte de los laboratorios farmacológicos, lo que originó la gran demanda de los trabajadores al comienzo de la campaña. Sin embargo en el año 2005 el "pico" se produce en la $3^{\mathrm{a}}$ semana, coincidiendo con la difusión en los medios de comunicación de noticias relacionadas con la posible pandemia de gripe aviar.

En los centros de trabajo del área 2, la cobertura vacunal ha sido similar en ambos años, excepto en el Hospital Universitario de la Princesa que en el año 2006 ha sido cercana al 50\%. Creemos que dicho aumento se debe a que se ofreció la vacuna directamente en el puesto de trabajo, facilitando su accesibilidad y evitando así que el trabajador tuviera que desplazarse al Servicio de Prevención. Por tanto acudir a vacunar a los puestos de trabajo es una estrategia a tener en cuenta en la próxima campaña de vacunación para incrementar la cobertura vacunal.

Respecto a la variable edad, se observa que aun no siendo significativa, los trabajadores vacunados en la campaña del 2006 son más jóvenes, esto podría tener relación con el aumento de estudiantes que se inocularon la vacuna en este año.

En cuanto a la distribución de las proporciones de vacunaciones en los distintos servicios, se observa una disminución significativa en Servicios Centrales, Administración y Hostelería en comparación con el año anterior respecto a los servicios médicos, considerada como basal, por lo que en siguientes campañas serían los servicios a priorizar.

En el año 2006, ha sido mayor la proporción de trabajadores que refieren haberse vacunado de la gripe el año anterior, (O.R. de 2.6 veces más), esto puede tener relación, a que en la campaña 2005 se demandaron más dosis por el temor a la pandemia de gripe aviar.

A la pregunta de si la vacuna de la gripe le produjo efectos secundarios en el año anterior, se observa que dichos efectos fueron menores en el año 2005 , es decir la vacuna produjo $2.38(1 / 0.42)$ veces menos reacciones que en el año 2004.

Uno de los grupos de población en los que está indicada la vacunación de la gripe, son aquellas personas que padezcan algún tipo de patología crónica. Al realizar esta pregunta en el 2005 declaran padecer enfermedad un $19 \%$, siendo en el 2006 un 23.5 $\%$. Esto contrasta con los trabajadores que refieren tomar medicación, un $30.5 \%$ y $27.1 \%$ respectivamente. Hay una diferencia significativa entre las personas que declaran padecer algún tipo de enfermedad y las que reciben medicación. A pesar de que existe una población femenina muy alta (71.6 $\%$ ) en las que la toma de anovulatorios es frecuente, no justifica estos hallazgos. Las cifras podrían reflejar el elevado consumo de medicamentos por parte de la población o la baja percepción de enfermedad.

Es interesante resaltar que en la Campaña 2006 el número de sanitarios vacunados ha sido menor $(\mathrm{O}: \mathrm{R}=2.08)$. Esto podría tener relación con la mayor información que los sanitarios poseen de las ventajas de la vacuna, pero sin olvidarse de los efectos secundarios (fiebre, malestar general, posible Guillain Barré, anafilaxia, angioedema, etc), sin tener en cuenta que la indicación principal, es el riesgo que suponemos como vehiculo de transmisión del virus entre los pacientes.

Por último, llama la atención entre ambas poblaciones la alta prevalencia de fumadores (alrededor del $25 \%$ ) a pesar de ser trabajadores que deberían estar más sensibilizados a los efectos perjudiciales del hábito tabáquico y que desde los últimos años se ha invertido en diversas actividades y consultas de deshabituación tabáquica dirigidas al público general y a los profesionales. 


\section{BIBLIOGRAFIA}

1. Campaña de vacunación antigripal y antineumocócica 2005. Dirección General de Salud Pública y Alimentación. Instituto de salud Pública. Septiembre de 2005.

2. Campaña de vacunación antigripal y antineumocócica 2006. Dirección General de Salud Pública y Alimentación. Instituto de salud Pública. Septiembre de 2006.

3. Bridges CB, Thompson WW, Meltzer MI Effectiveness and cost-benefit of influenza vaccination of healthy working adults: A randomized controlled trial. JAMA. 2000 Oct 4; 284(13):1655-63.

4. Wilde JA, McMillan JA, Serwint J. Effectiveness of influenza vaccine in health care professionals: a randomized trial. JAMA. 1999; 281:908-913.

5. Saxén H, Virtanen M. Randomized, placebo-controlled double blind study on the efficacy of influenza immunization on absenteeism of health care workers. Pediatr Infect Dis J. 1999 Sep;18 (9):779-83.

6. Recommended composition of influenza virus vaccines for use in the 2006-2007 influenza season. Wkly Epidemiol, nº 9, 3 march 2006. 